UCRL-JC-122248

PREPRINT

\title{
The Safety-Critical Software Evaluation Assistant (SEA)
}

\author{
Warren L. Persons
}

This paper was prepared for submittal to the Second International Federation of Automatic Control (IFAC) Workshop on Safety and Reliability in Emerging Control Technologies

Daytona Beach, Florida

November 1-3, 1995

\section{October 1995}

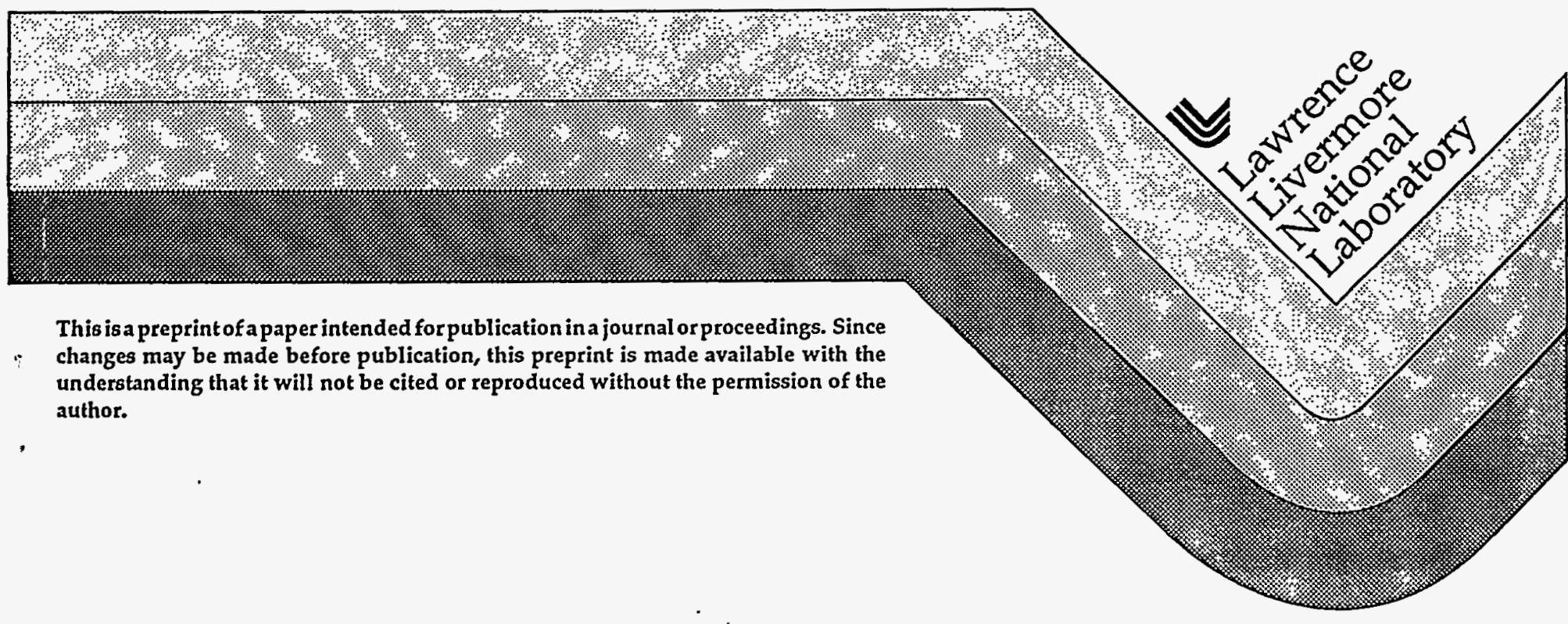

This is a preprint of a paper intended forpublication in a journal or proceedings. Since changes may be made before publication, this preprint is made available with the understanding that it will not be cited or reproduced without the permission of the author. 


\section{Disclaimer}

This document was prepared as an account of work sponsored by an agency of the United States Government. Neither the United States Government nor the University of California, nor any of their employees, makes any warranty, express or implied, or assumes any legal liability or responsibility for the accuracy, completeness, or usefulness of any information, apparatus, product, or process disclosed, or represents that its use would not infringe privately owned rights. Reference herein to any specific commercial product, process, or service by trade name, trademark, manufacturer, or otherwise, does not necessarily constitute or imply its endorsement, recommendation, or favoring by the United States Government or the University of California. The views and opinions of authors expressed herein do not necessarily state or reflect those of the United States Government or the University of California.

This work was supported by the United States Nuclear Regulatory commission under a Memorandum of Understanding with the United States Department of Energy, and performed under the auspices of the U.S. Department of Energy by Lawrence Livermore National Laboratory under Contract W-7405-Eng-48. 


\title{
THE SAFETY-CRITICAL SOFTWARE EVALUATION ASSISTANT (SEA)*
}

\author{
Warren L. Persons \\ University of California \\ Computer Safety \& Reliability Group \\ Fission Energy and Systems Safety Program \\ Lawrence Livermore National Laboratory \\ 7000 East Avenue, L-632 \\ Livermore, CA 94550 \\ E-mail: persons2@llnl.gov
}

\begin{abstract}
The Computer Safety and Reliability Group at Lawrence Livermore National Laboratory (LLNL) is researching the evaluation of software used in safety-critical applications. This paper describes one of the research and development efforts currently underway to model the software evaluation process and to develop a software evaluation tool. One of the primary techniques available for determining the safety of software proposed for use in safety-critical applications is to evaluate the software development process and the resulting products. This model of the evaluation process was influenced by several factorsthe underlying motivation was to identify, control and reduce the risk inherent in building safety-critical software systems. This prototype tool, the Software Evaluation Assistant (SEA), assists and guides evaluators as they analyze safety-critical software. SEA describes specific evaluation goals, provides a brief overview of the specific evaluation process, identifies potential risks of not performing the evaluation, identifies the skills required to carry out the evaluation of a particular topic, identifies the material that should typically be available for the evaluation, and poses questions used to examine and rate the software item.
\end{abstract}

Keywords: Artificial Intelligence (AI), Case-Based Reasoning (CBR), Evaluation, Knowledge Base, Knowledge-based system, Safety-Critical, Software Safety.

\subsection{INTRODUCTION}

Today, software components are used in safetyrelated and safety-critical systems and this fact presents significant challenges to software developers, evaluators, and sponsors as they attempt to reduce potential hazards associated with these systems. These challenges are faced by the United States Department of Energy (DOE), the Food and Drug Administration (FDA), the Department of Transportation (DOT), the Federal Aviation Administration (FAA), the Department of Defense (DoD), the Nuclear Regulatory Commission (NRC) and private corporations and contractors who provide software and software services to these organizations.
Various risks are inherent in the complex process of producing high-quality, safety-critical software. An overview of risk identification, management and mitigation is presented in Charette (1989). Additional concerns regarding safety-critical software include the fact that (1) more and more system functions (safety and otherwise) are being allocated to software components, (2) the software development practices used to develop this safety-critical software are not as thorough as many would desire, (3) software development practices are seldom based on generally accepted software engineering principles, and (4) development practices seldom support effective software verification and validation (V\&V) activities.

*UCRL-JC-122248. This work was performed under the auspices of the U.S. Department of Energy by Lawrence Livermore National Laboratory under contract no. W-7405-Eng-48. 
The real need is a technique to help establish sufficient trust in safety-critical systems in a costefficient fashion. Lawrence Livermore National Laboratory's research provides one approach to evaluating safety-critical software that is risk-driven and uses national and international consensus software development standards as a basis for the evaluation. Details of this evaluation process are described in Persons (1994). This paper describes work in progress in moving from a proof-of-concept prototype to a fully functional tool, the Software Evaluation Assistant (SEA). SEA incorporates techniques and knowledge from the disciplines of computer science, artificial intelligence, and software engineering and is designed for an evaluation team to analyze a vendor's software development practices.

\subsection{BACKGROUND}

The evaluation process supported by SEA focuses on three major thrust areas: software development planning activities, the quality of interim software products, and the software development process used to produce the products. SEA supports the evaluation team by permitting a customized evaluation process, providing preliminary indications of the "goodness" of these software products, and suggesting where potential problems may exist.

The knowledge and guidance used to create the prototype software tool is based on existing software development standards and generally accepted software engineering principles. SEA provides (1) the capability to create and use a historical, experiential database to interpret and analyze the current evaluation situation, (2) information needed to guide the evaluation team members as they perform their evaluation, and (3) specific recommendations that can help the software development team reduce potential safety hazards and improve the development process.

\subsection{THE SEA EVALUATION MODEL}

\subsection{SEA Philosophy}

There are two general methods used by leading-edge software development companies to increase the trustworthiness of safety-critical software: (1) reduce the number of errors introduced during the software development process, and (2) increase the percentage of overall errors found prior to system installation. The goal of the first approach is producing "error free software" by implementing continuous process improvements to essentially eliminate errors before they are introduced. The second approach uses traditional quality-affecting practices such as inspections, reviews, and verification and validation (V\&V) activities espoused by various national and international software development standards from
ANSI, IEEE, ISO, IEC, etc. (IEC JTC1-SC7, IEEE 1012-1986, IEEE 1074-1991)

The underlying purpose of software evaluation is"to $=4: 4 ; 1, \cdots$. help identify, assess and manage software usage risks. More specifically, SEA supports and enables the evaluation team as they strive to achieve the following goals for safety-critical software (adapted from IEEE Standard 1012): (1) increase knowledge of the software development process, (2) detect and correct errors as early as possible in the development effort, (3) lessen the chances of cost and schedule overruns during development, (4) enhance software quality and reliability, and (5) quickly assess the consequences of proposed changes to the software.

The development of the SEA prototype was guided by: (1) the need for consistent evaluations across evaluation teams and over time and (2) the goal to support evaluations throughout the entire development process. SEA supports two separate types of safety-critical software evaluations. The first type of evaluation, referred to as a screening evaluation, is a filter that increases confidence that the software vendor is addressing many of the qualities typically associated with high-integrity, trusted, safety-critical software. If the screening evaluation is successful, the vendor's activity, process, or product is subjected to a detailed evaluation to examine additional factors that should be present in safety-critical systems. The detailed evaluation process supported by SEA is described in Persons (1994).

\subsection{Software Life Cycle Activities}

For the purposes of this paper, the ultimate result of the software development process is an application that runs on a computer as part of a safety-critical system and possesses attributes deemed desirable by the user, sponsor, and/or developer (e.g., safety, reliability, performance, usability and specified functions). Since many life cycles have been proposed for developing software, SEA's support of the evaluation process can be adapted to a particular software vendor's software-development practices.

Each software life cycle typically contains similar activities; however, they generally differ in when (and how) specific activities are performed. No particular life cycle is assumed when using SEA, but it is presumed that activities within the developer's life cycle yield interim software products, each of which can be evaluated by SEA. The eight software development activities shown in Figure 1 will be assumed in this paper: (1) Planning (and evaluation), (2) Requirements (and evaluation), (3).Design (and evaluation), (4) Implementation (and evaluation), (5) Integration (and evaluation), (6) Validation (and evaluation), (7) Installation (and evaluation), and (8) Operation and maintenance. 
Figure 1 suggests several points at which specific SEA evaluations can occur. Many reliability, performance, and safety problems can only be resolved by careful design of the software product, so these (and other) properties should be evaluated early in the software life cycle, no matter which life cycle is used by the vendor. Additional information can be found in Lawrence (1993).

\subsection{The SEA Evaluation Process}

The SEA evaluation process is applicable to any software development activity, process, or product created as part of the software development effort. There are a number of purposes for evaluating a vendor's software processes or products. A primary purpose is to assess the risk of software failure. A secondary goal is to determine the overall quality of the software and collect convincing evidence to support the evaluation. The foundation of an effective evaluation is a process that defines and measures the effectiveness of each safety-critical software development activity. SEA is based upon a hierarchical model whose evaluation properties are generally recognized by the software engineering community as items necessary, but not sufficient, to produce quality software products. Experience suggests that evaluation of these properties, expert judgment, and independent evaluation remain necessary to increase the confidence placed in safetycritical software.

The screening and detailed evaluations identify potential problems and improvements required before the software products appear as deliverable items. One very important point is that much of the benefit of the evaluation process is rooted in the analysis and thinking processes of the evaluators as they use SEA to perform an evaluation. The detailed evaluation should be performed for software items receiving a "go" rating from the screening evaluation. One can think of the detailed evaluation process as an analysis of a software item that has been generally given a "preliminary clean bill of health" or "go" rating by the screening evaluation process. The detailed process confirms or rejects the preliminary screening rating. Both screening and detailed evaluations should be applied to each item under consideration that is part of a safety-critical system. A top-level evaluation process flow is shown in Figure 2.

\subsection{SEA PROTOTYPE OVERVIEW}

SEA provides computer assistance to the safetycritical software evaluation process. Task analysis studies suggest that to be a viable tool, SEA should: (1) help manage evaluation process complexity, (2) define activity-specific evaluation procedures, (3) create evaluation specific criteria and guidance, (4) use consistent evaluation practices, and (5) interact with the evaluator through a common graphical user interlace. The SEA prototype demonstrated that the integration of evaluators, evaluation methods, and domain knowledge is feasible through an automated tool. SEA's design goals are to:

- increase evaluation accuracy, consistency and productivity

- support customization of the evaluation process

- base the evaluation on industry standards and consensus software engineering principles

- reduce the time and effort involved in an evaluation

- maintain a database of historical information

- provide multiple levels of guidance and help

- provide security and access controls to safeguard evaluation data

To achieve these goals, SEA encapsulates knowledge of software development processes, product assurance and V\&V techniques, and evaluation practices. SEA provides assistance in documenting the evaluation, guidance on the use of standards and detailed evaluation criteria, and a rating process with suggested interpretations. A conceptual architecture for SEA is shown in Figure 3.

SEA is based on artificial intelligence concepts common to machine problem solving techniques and uses a knowledge base that contains facts, heuristics and rules believed to be associated with good software development practices. SEA's architecture: accommodates new facts; accommodates beliefs and cases as they evolve; supports various types of reasoning and uses this information to make judgments about the software products and properties associated with them; provides explanations on how judgments were made; and suggests corrective actions to improve items being examined.

The SEA prototype is a Microsoft Windows application running on an IBM-compatible laptop computer. The prototype was developed using an object-oriented paradigm and a development shell that supports Case-Based Reasoning, fuzzy logic, and rule-based inferencing. The prototype provides the evaluator with the capability to access historical data, applicable standards and guidance during the evaluation process which can be used to identify. trends and help evaluators focus on the aspects of the safety-critical software development process that typically present greater risk.

\subsection{CONCLUSION}

This paper presents a description of a proof-ofconcept prototype of a safety-critical software evaluation model. Preliminary research suggests that automation of an evaluation process model is feasible using existing technology. The SEA prototype has shown that many of the goals for the safety-critical Software Evaluation Assistant are realizable using standard AI problem-solving techniques. In particular, the work in progress has shown that the 
Case-Based Reasoning paradigm can be effectively used to analyze a new evaluation situation based on comparisons and adaptations of stored cases that are 'pär of SEA's experience base (case-base). (Kolodner 1993; Riesbeck 1989; Schank 1994)

However, much work remains to be done in the analysis and trade-offs associated with alternative evaluation approaches. Future plans include the investigation of alternative problem-solving strategies, the use of additional rule-based inferencing, expanded use of the Case-Based Reasoning, proof of feasibility of using a fuzzy-logicbased evaluation process, and the exploration of Artificial Neural Network modeling of the evaluation rating processes used by SEA.

\subsection{REFERENCES}

Charette, Robert N. 1989. Software Engineering Risk Analysis and Management. McGraw-Hill.

IEC JTC1-SC7. 1992. Information Technology Software Life-Cycle Process. IEC ISO
Committee Draft 12207, IEC(JTC1)-SC7, August.

IEEE 1012-1986. IEEE Standard for Software Verification and Validation Plans.

IEEE 1074-1991. IEEE Standard for Developing Life Cycle Processes.

Kolodner, Janet L. 1993. Case-Based Reasoning. Morgan Kaufmann Publishers, Inc.

Lawrence, J. D, 1993. Software Reliability and Safety in Nuclear Reactor Protection Systems. Lawrence Livermore National Laboratory. Nuclear Regulatory Commission Report NUREG/CR 6101, UCRL-ID-114839, November.

Persons, W. L. and J. D. Lawrence. 1994. Assessing Safety-Critical Software in Nuclear Power Plants. Lawrence Livermore National Laboratory. Draft Internal Report, April.

Riesbeck, Christopher K. and Roger C. Schank. 1989. Inside Case-Based Reasoning. Lawrence Erlbaum Associates, Inc.

Schank, Roger C., Alex Kass, and Christopher K. Riesbeck. 1994. Inside Case-Based Explanation. Lawrence Erlbaum Associates, Inc.

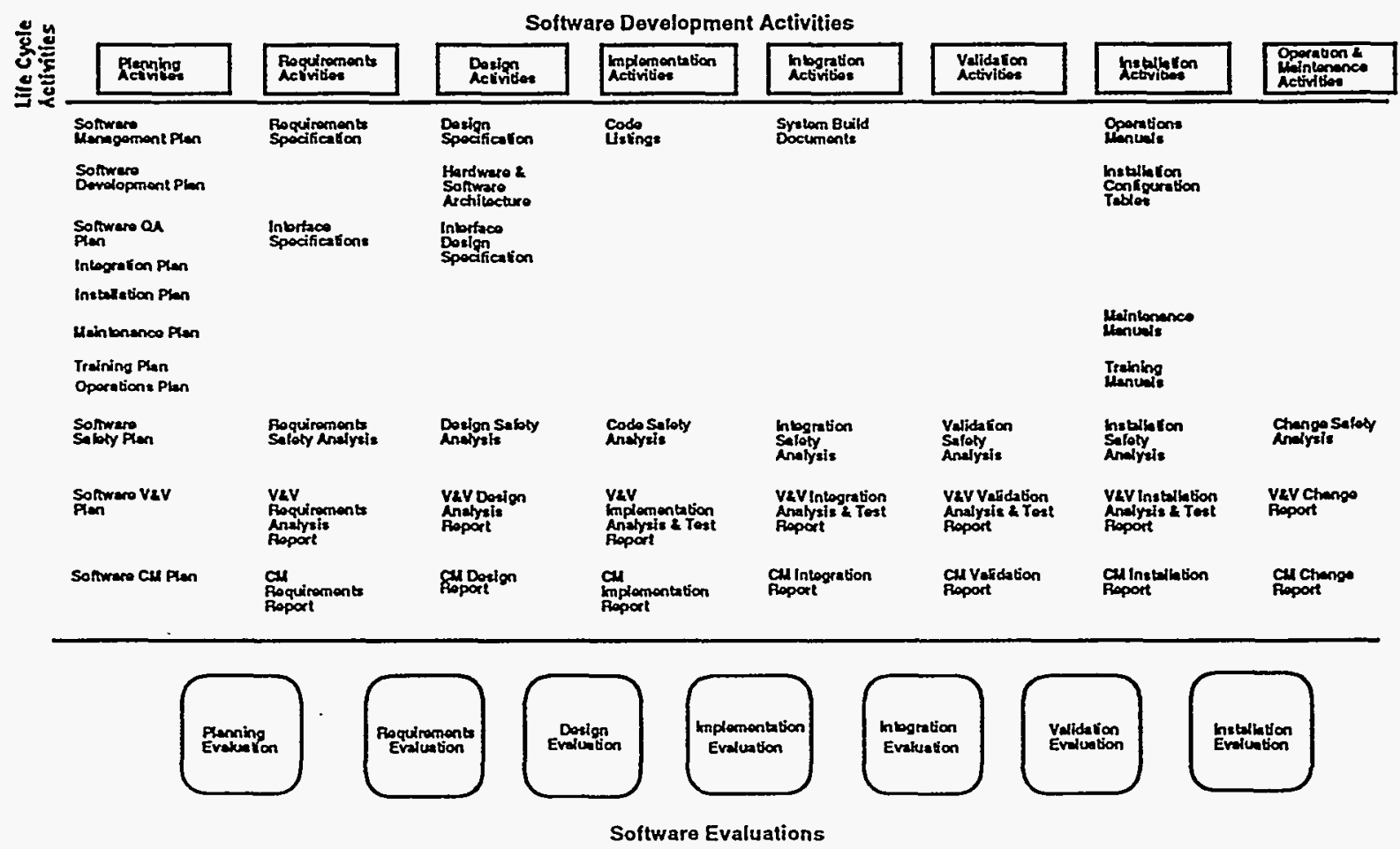

Figure 1. Software Activities and Evaluations Supported by SEA 


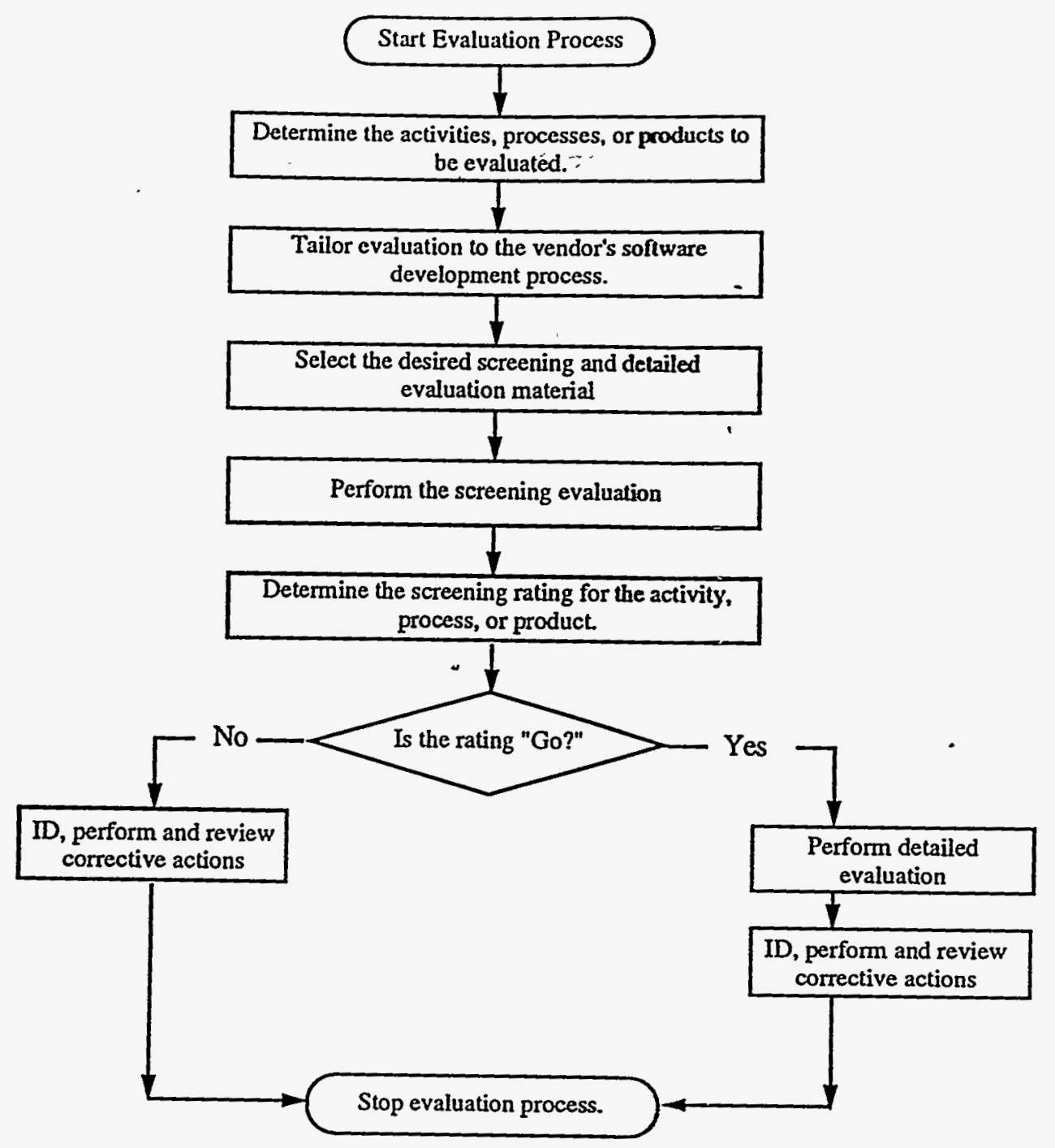

Figure 2. SEA Evaluation Process Flow 


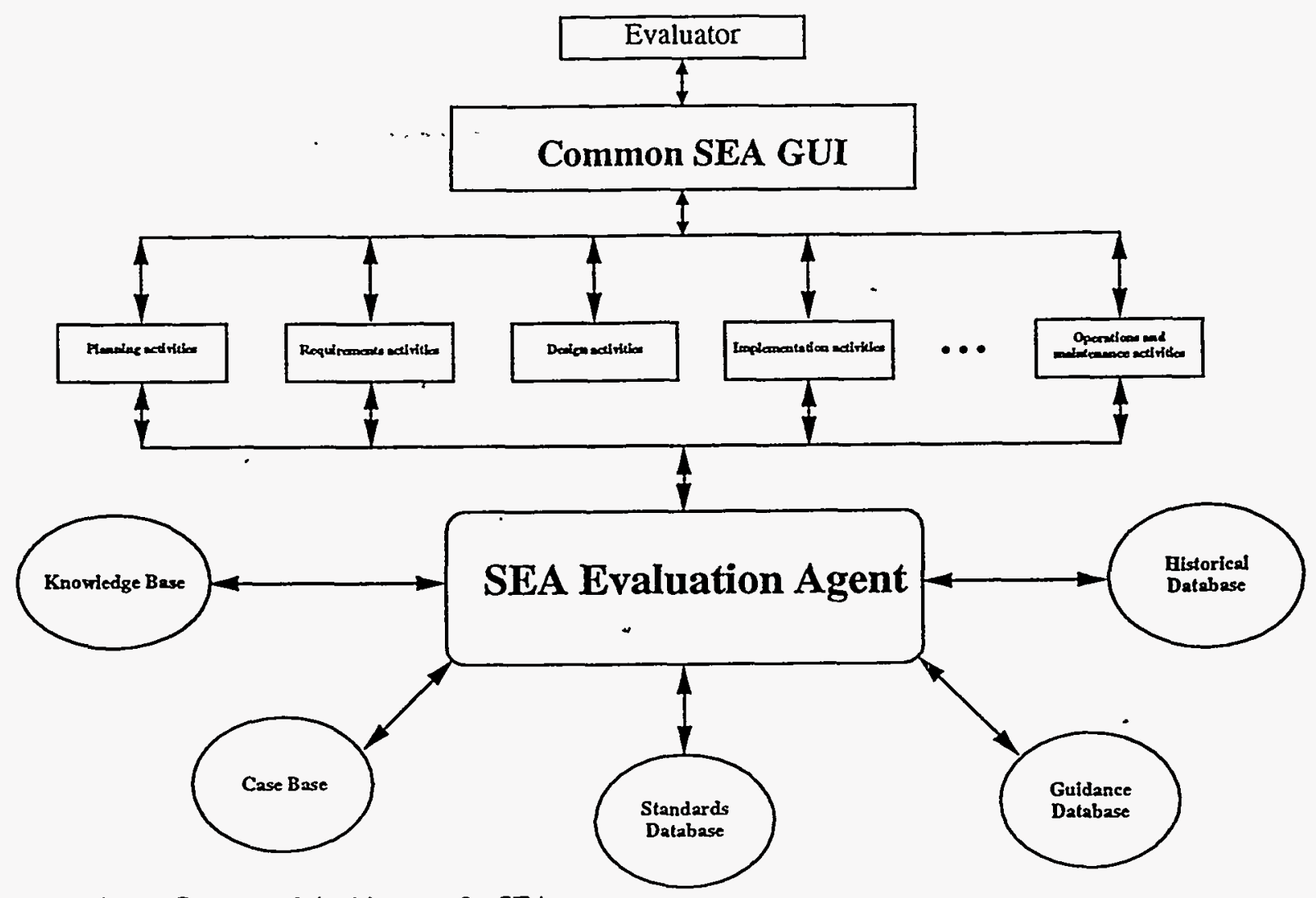

Figure 3. Conceptual Architecture for SEA 
